\title{
Acknowledgements to Referees
}

The editors wish to express their sincere appreciation to the numerous colleagues who have critically assessed submitted papers thereby providing the information indispensable for maintaining the scientist standing of Caries Research. J.M. ten Cate

M. Addy

J.K. Ainamo

R.J. Anderson

B. Angmar-Månsson

J. Arends

R.E. Baier

F.H. Barbakow

D. Beighton

B. Bibby

R.J. Billings

J. Birkeland

D. Birkhed

J. de Boever

J.M.P.M. Borggreven

J.J. ten Bosch

A. Boyde G.H.W. Bowden W.H. Bowen P. Brandtzaeg D. Bratthall A.L.J.J. Bronckers A.T. Brown J. Brunelle

C. Bruun

J.J. Burckhardt J.P. Carlos J. Carlsson V. Caslavska S.J. Challacombe N.W. Chilton B.H. Clarkson J.J. Clarkson R.E. Corpron S.L. Creanor M.E.J. Curzon

C. Dawes

D.P. DePaola

G.H. Dibdin

W. Distler

M.C. Downer

F.C.M. Driessens

D. Drucker

R. Duckworth

R. Duguid

H. Duschner A.G. Dijkman J.E. Eastoe W.M. Edgar S. Edwardsson

A.D. Eisenberg J. Ekstrand R.J. Elderton J.C. Elliott C-G. Emilson Th. Ericson J.D.B.

Featherstone F.R. von der Fehr O. Fejerskov E.D. Fillery A.R. Firestone R.J. Fitzgerald R. Frank R.J. Gibbons P.E. Gjermo P.O. Glantz R.L. Glass H. Graf

E.J.’s-Gravenmade R.M. Green T.H. Grenby 

A. Groeneveld
P. Gron
B. Guggenheim

I.R. Hamilton

P.S. Handley

J.L. Hardwick

T. Hargreaves

D.I. Hay

M. Hayes

A. Hefti

J.D. Hillman

J.S. van der Hoeven

W.P. Holbrook

P.J. Holloway

P. Hoppenbrouwers

H.S. Horowitz

P. Hotz

J. van Houte

B. Houwink

T. Imfeld

G.S. Ingram

G.N. Jenkins

M.E. Jensen

N.W. Johnson

C.Jones

M.H.deJong

W.L. Jongebloed

M. Joost Larsen

S. Joyston-Bechal H. Kalsbeek S. Kashket C.W. Keevil E.A.M. Kidd M. Kilian

E. Kirkegaard

I. Kleinberg

B. Köhler

K.G. König

T. Koulourides W. Künzel K.P. Lang S.A. Leach T. Lehner

A. Linde

H. Löe

W.J. Loesche

C. van Loveren

H. Luoma

K.K. Mäkinen

F. Manji

H.C. Margolis

R.E. Marquis

T.M. Marthaler

B.C. McBride

J.M. Mellberg 
J.H. Meurman

S. Michalek

L. Mikkelsen F.H.M. Mikx CM. Mitropoulos T. Modéer E.C. Moreno S. Mundorff J.J. Murray G.H. Nancollas J.M. Navia M.N. Naylor D.G.A. Nelson E. Newbrun H. Newesely H.N.

Newman G.S. Nixon J. Norén

B. Nyvad

D.M. O’Mullane

E.I.F. Pearce

Ch. Penning

L.G Petersson D.F.G. Poole S. Poulsen D.J. Purdell-Lewis H.R. Rawls D.H. Retief A.H. Rogers G Rølla A.J. Rugg-Gunn R.R.B. Russe A. Saxto

M.J.M. Schaeken A.A. Scheie A. Sheiham R. Schmid M.H. Schoen H.E. Schroeder L. Seppå

L.M. Silverstone T. Sønju C.J. Spak P.F. van der Stelt K.W. Stephen G.K. Stookey R. Strang M.

Svanberg J.M. Tanzer A. Tatevossian J.O. Tenovuo

E. Theilade

A. Thylstrup

R.Z.M.Triller

G.J. Truin

F. Vogels

M. Vulli

W. Wade

BJ. Wagg

G.J. Walker

J.A. Weatherell

A.H. Weerkamp

J.S. Wefel

J. Wennstrom G.M. Whitford R.A.D. William M. Wilson J.M. Wilton GB. Winter S.C. Ziesenitz 